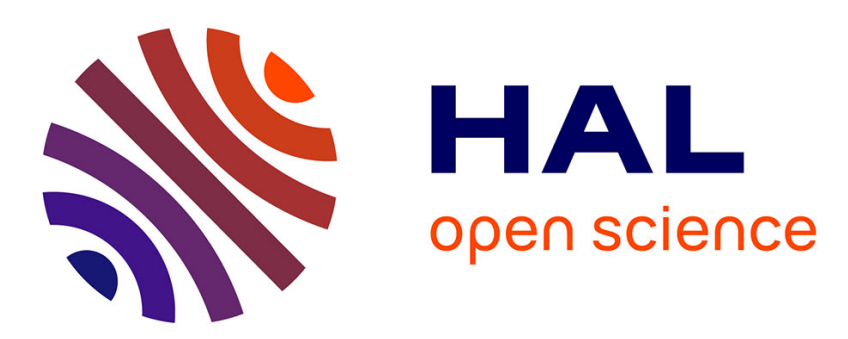

\title{
A generalization of the Random Energy Model which includes correlations between energies
}

\author{
B. Derrida
}

\section{To cite this version:}

B. Derrida. A generalization of the Random Energy Model which includes correlations between energies. Journal de Physique Lettres, 1985, 46 (9), pp.401-407. 10.1051/jphyslet:01985004609040100 . jpa-00232535

\section{HAL Id: jpa-00232535 https://hal.science/jpa-00232535}

Submitted on 1 Jan 1985

HAL is a multi-disciplinary open access archive for the deposit and dissemination of scientific research documents, whether they are published or not. The documents may come from teaching and research institutions in France or abroad, or from public or private research centers.
L'archive ouverte pluridisciplinaire HAL, est destinée au dépôt et à la diffusion de documents scientifiques de niveau recherche, publiés ou non, émanant des établissements d'enseignement et de recherche français ou étrangers, des laboratoires publics ou privés. 


\title{
LE JOURNAL DE PHYSIQUE-LETTRES
}

J. Physique Lett. 46 (1985) L-401 - L-407

1er MAI 1985, PAGE L-401

Classification

Physics Abstracts

$05.50-64.60 \mathrm{C}-75.50 \mathrm{~K}$

\section{A generalization of the Random Energy Model which includes correlations between energies}

\author{
B. Derrida \\ Service de Physique Théorique, CEN-Saclay, 91191 Gif-sur-Yvette Cedex, France
}

(Reçu le 18 février 1985, accepté le 18 mars 1985)

\begin{abstract}
Résumé. - Le modèle d'énergies aléatoires est généralisé pour prendre en compte des corrélations arbitraires entre paires de niveaux d'énergies. Ce modèle reste un modèle de verre de spin soluble. Le gel brutal du modèle à énergies aléatoires peut être remplacé en gel progressif quand on baisse la température.
\end{abstract}

\begin{abstract}
The Random Energy Model is generalized to treat arbitrary correlations between pairs of energy levels. This Generalized Random Energy Model remains soluble. The sudden freezing which occurs in the RE model can, in the generalized version, become a progressive freezing when temperature decreases.
\end{abstract}

The mean field theory of spin glasses has been, in the last ten years, a subject of great activity [1]. Among the numerous approaches toward the solution of the Sherrington-Kirkpatrick (SK) model [2], a simple one led to the introduction of a new model [3, 4] which could be solved exactly : the Random Energy Model (REM) [5-8]. This model can be considered as an extreme simplification of spin glass models. The main simplification in the REM was that the energies $E_{1}$ and $E_{2}$ of two different configurations are not correlated. For more realistic models, like the SK model, the $p$ spin models introduced in references [3] and [4] or spin glass models in finite dimension, one knows that the energies $E_{1}$ and $E_{2}$ of 2 different configurations are in general correlated. In some cases one can calculate these correlations. Thus a very important question is to ask whether the RE model can be considered as the zeroth order approximation of a scheme which would be able to treat these correlations.

The purpose of the present work is to generalize the RE model in such a way that any correlation between pairs of energies can be taken into account.

The Generalized Random Energy Model (GREM) is defined in the following way. One considers a system of $2^{N}$ configurations : configuration $v$ has an energy $E_{v}\left(1 \leqslant v \leqslant 2^{N}\right)$. These configurations are grouped according to a very simple hierarchy of $n$ levels. Let us first group 
the $2^{N}$ configurations into groups of $\left(\alpha_{n}\right)^{N}$ configurations each : there are of course $\left(2 / \alpha_{n}\right)^{N}$ such groups. We shall say that at the $n$th level of the hierarchy, we have $\left(2 / \alpha_{n}\right)^{N}$ groups of $\left(\alpha_{n}\right)^{N}$ configurations. At the next level (level $n-1$ of the hierarchy), we group again these groups : each group at the $(n-1)$ th level contains $\left(\alpha_{n-1}\right)^{N}$ groups at level $n$. Therefore at level $n-1$ of the hierarchy, one has $\left[2 /\left(\alpha_{n} \alpha_{n-1}\right)\right]^{N}$ groups of $\left(\alpha_{n} \alpha_{n-1}\right)^{N}$ configurations each. One can repeat this grouping procedure. At the $i$ th level, there are $\left[2 /\left(\alpha_{i} \ldots \alpha_{n}\right)\right]^{N}$ groups of $\left(\alpha_{i} \alpha_{i+1} \ldots \alpha_{n}\right)^{N}$ configurations each. Clearly since there are altogether $2^{N}$ levels, one must have

$$
\log \alpha_{1}+\log \alpha_{2}+\cdots+\log \alpha_{n}=\log 2 \text {. }
$$

The hierarchy constructed in this way is very reminiscent of the ultrametric structure [9] (see Fig. 1).

We have now to say how the values of the $2^{N}$ energies $E$ are chosen. By definition of the model, $E_{v}$ is given by a sum of $n$ random numbers

$$
E_{v}=\varepsilon_{1}^{(v)}+\varepsilon_{2}^{(v)}+\cdots+\varepsilon_{n}^{(v)}
$$



Fig. 1. - The grouping of configurations in the Generalized Random Energy Model. Here $n=3$ and $\alpha_{1}^{N}=2, \alpha_{2}^{N}=2, \alpha_{3}^{N}=4$.

where $\varepsilon_{i}^{(v)}$ is a random number distributed according to a given distribution $\rho_{i}$ that we choose for simplicity to be a Gaussian.

$$
\rho_{i}\left(\varepsilon_{i}\right)=\frac{1}{\sqrt{\pi N J^{2} a_{i}}} \exp \left(-\frac{\varepsilon_{i}^{2}}{N J^{2} a_{i}}\right) .
$$

We shall say that $\varepsilon_{i}^{(v)}$ is the contribution to $E_{v}$ coming from the $i$ th level of the hierarchy. Note that the width of the distribution depends on $i$. By definition of the model, two energies $E_{v}$ and $E_{\mu}$ which belong to the same group at the $i$ th level of the hierarchy have the same $\varepsilon_{j}$ for $j \leqslant i-1$ but have different $\varepsilon_{j}$ for $j \geqslant i$ if they belong to different groups at the $(i+1)$ th level.

$$
\begin{aligned}
& \varepsilon_{j}^{(v)}=\varepsilon_{j}^{(\mu)} \text { for } j \leqslant i-1 \\
& \varepsilon_{j}^{(v)} \neq \varepsilon_{j}^{(\mu)} \text { for } j \geqslant i .
\end{aligned}
$$

When the $\varepsilon_{j}^{(v)}$ and $\varepsilon_{j}^{(\mu)}$ are different, they are independent. Also $\varepsilon_{j}^{(v)}$ and $\varepsilon_{k}^{(\mu)}$ are always independent for $j \neq k$.

Thus we see that the model is defined once the two sequences $\alpha_{i}$ and $a_{i}$ are given for $1 \leqslant i \leqslant n$. To normalize the distribution, we shall impose the following condition

$$
\sum_{i=1}^{n} a_{i}=1
$$


Let us now describe the correlations between the energies of the configurations. First we can calculate the probability $P(E)$ that a given configuration $v$ has an energy $E$. Clearly since $E_{v}$ is a sum of independent Gaussian random variables (see Eqs. (2), (3) and (5)) one has

$$
P(E)=\frac{1}{\sqrt{\pi N J^{2}}} \exp \left(-\frac{E^{2}}{N J^{2}}\right) .
$$

We can also calculate $P_{i}\left(E_{1}, E_{2}\right)$, the probability that configurations $v$ and $\mu$ have respectively energies $E_{1}$ and $E_{2}$. This probability distribution will obviously depend on the distance between the 2 configurations in the hierarchy. If we call $i$ the highest level for which $v$ and $\mu$ belong to the same group, one can write

$$
\begin{aligned}
& E_{1}=\phi+\phi_{1} \\
& E_{2}=\phi+\phi_{2}
\end{aligned}
$$

where

$$
\phi=\sum_{j=1}^{i-1} \varepsilon_{j}^{(v)}, \quad \phi_{1}=\sum_{j=i}^{n} \varepsilon_{j}^{(v)}, \quad \phi_{2}=\sum_{j=i}^{n} \varepsilon_{j}^{(\mu)}
$$

$\phi, \phi_{1}$ and $\phi_{2}$ are random independent Gaussian variables whose widths are known. Therefore $P_{i}\left(E_{1}, E_{2}\right)$ is given by

$$
\begin{aligned}
P_{i}\left(E_{1}, E_{2}\right) & =\text { const. } \times \int \mathrm{d} \phi \exp \left[-\frac{\left(E_{1}-\phi\right)^{2}+\left(E_{2}-\phi\right)^{2}}{N(1-v) J^{2}}-\frac{\phi^{2}}{N v J^{2}}\right] \\
& =\text { const. } \times \exp \left[-\frac{\left(E_{1}+E_{2}\right)^{2}}{2 N(1+v) J^{2}}-\frac{\left(E_{1}-E_{2}\right)^{2}}{2 N(1-v) J^{2}}\right]
\end{aligned}
$$

where

$$
v=\sum_{j=1}^{i-1} a_{j}
$$

Given a configuration $(v)$, the number $\mathrm{e}^{N u}$ of configurations $(\mu)$ to which formula (10) applies is just the number of configurations which belong to the same group at level $i$ and to different groups at level $i+1$

$$
\mathrm{e}^{N u}=\left[\left(\alpha_{i}\right)^{N}-1\right]\left(\alpha_{i+1} \ldots \alpha_{n}\right)^{N}
$$

Clearly for $N \rightarrow \infty$, one has

$$
u=\sum_{j=i}^{n} \log \alpha_{j}
$$

One sees that by choosing the $a_{i}$ and $\alpha_{i}$, one can produce any probability distribution $P_{i}\left(E_{1}, E_{2}\right)$. In the limit $n \rightarrow \infty$, this Generalized Random Energy Model (GREM) is just defined by the relationship between $u$ and $v: v$ is a decreasing function of $u$ which passes through $(u=0, v=1)$ and $(u=\log 2, v=0)$.

One can build a GREM which has exactly the same $P\left(E_{1}, E_{2}\right)$ as a given spin glass model. For example, for the $p$ spin models defined in references [3-5] and for the SK model (which is the case $p=2$ ) it has been shown that for 2 different configurations which have $N x$ identical spins, the probability distribution $P_{x}\left(E_{1}, E_{2}\right)$ depends on $x$ and has exactly the form (10) (see Eq. (6) of Ref. [4]) provided one takes

$$
v=(2 x-1)^{p}
$$


In these spin models, one knows that given a configuration $v$, the number $\mathrm{e}^{N u}$ of configurations $\mu$ which have $N x$ spins identical with configuration $v$ is

$$
\mathrm{e}^{N u}=\frac{N !}{(N x) !(N(1-x)) !}
$$

Therefore for large $N$, one has

$$
u=-x \log x-(1-x) \log (1-x)
$$

Thus a GREM whose $u$ and $v$ are related by (14) and (15) ( $x$ varying between 1 and $1 / 2)$ has exactly the same correlations between pairs of energies as the corresponding $p$ spin glass model. A consequence is that the GREM and the $p$ spin glass model have exactly the same $\overline{Z(T)}$ and the same $\overline{Z\left(T_{1}\right) Z\left(T_{2}\right)}$ for any temperature $T_{1}$ and $T_{2}$.

Here $Z(T)$ denotes the partition function at temperature $T$ and the bar means the average over disorder. It is also clear that there is no reason a priori that $\overline{Z\left(T_{1}\right) Z\left(T_{2}\right) Z\left(T_{3}\right)}$ would be identical in the two models.

In the last part of this work, I would like to explain the mechanism which allows the GREM to be solved exactly for any finite $n$ and for any choice of the $\alpha_{i}$ and $a_{i}$. As $n$ increases the solution becomes more and more complicated (at least for arbitrary $\alpha_{i}$ and $a_{i}$ ) but can always be done explicitly. I shall only describe here the solution for $n=2$ and indicate how one can continue for larger $n$.

For $n=1$, equations (1) and (5) impose that $\alpha_{1}=2$ and $a_{1}=1$ and the GREM reduces to the RE model whose solution is known [3-7].

For $n=2$, we shall see that the GREM has already more structure than the RE model. The solution is a simple generalization of the entropy argument which was used to solve the RE model.

For $n=2$, the system consists of $\left(\alpha_{1}\right)^{N}$ groups of $\left(\alpha_{2}\right)^{N}$ configurations each. One has of course

$$
\log \alpha_{1}+\log \alpha_{2}=\log 2 \text {. }
$$

For each group, one has to choose a random number $\varepsilon$ according to a distribution $\rho_{1}(\varepsilon)$

$$
\rho_{1}(\varepsilon)=\frac{1}{\sqrt{\pi N J^{2} a_{1}}} \exp \left(-\frac{\varepsilon^{2}}{N J^{2} a_{1}}\right) .
$$

Then, once $\varepsilon$ has been chosen for a given group, each energy $E$ of that group must be chosen according to the distribution $\tilde{\rho}(E, \varepsilon)$ given by

$$
\tilde{\rho}(E, \varepsilon)=\frac{1}{\sqrt{\pi N J^{2} a_{2}}} \exp \left(-\frac{(E-\varepsilon)^{2}}{N J^{2} a_{2}}\right) .
$$

The constraint (5) becomes here

$$
a_{1}+a_{2}=1
$$

Let us estimate the number $\mathcal{N}_{1}(\varepsilon)$ of groups having an energy $\varepsilon$. Like in the RE model, one has

$$
\begin{array}{ll}
\mathcal{N}_{1}(\varepsilon) \sim \overline{\mathcal{N}_{1}(\varepsilon)} \sim \exp N\left(\log \alpha_{1}-\frac{1}{a_{1}}\left(\frac{\varepsilon}{N J}\right)^{2}\right) & \text { if } \overline{\mathcal{N}_{1}(\varepsilon)} \gg 1 \\
\mathcal{N}_{1}(\varepsilon) \sim 0 & \text { if } \overline{\mathcal{N}_{1}(\varepsilon)} \ll 1 .
\end{array}
$$


The typical value of $\mathcal{N}_{1}(\varepsilon)$ is equal to its average when the average is large and is zero when the average is small $[3,4]$ i.e. $|\varepsilon|>N J \sqrt{a_{1} \log \alpha_{1}}$. Now that we know $\mathcal{N}_{1}(\varepsilon)$, we can calculate the typical value of the number $\mathcal{N}_{2}(E)$ of configurations having energy $E$. Let us define $Q_{2}(E)$ by

$$
Q_{2}(E) \sim \int \mathcal{N}_{1}(\varepsilon) \mathrm{d} \varepsilon \exp N\left(\log \alpha_{2}-\frac{1}{a_{2}}\left(\frac{E-\varepsilon}{N J}\right)^{2}\right)
$$

Since $\mathcal{N}_{1}(\varepsilon)=0$ if $|\varepsilon|>N J \sqrt{a_{1} \log \alpha_{1}}$, the integral in (21). has to be carried out on the interval $|\varepsilon|<N J \sqrt{a_{1} \log \alpha_{1}}$. Again one can repeat the argument to calculate the typical value of $\mathcal{N}_{2}(E)$.

$$
\begin{array}{cccc}
\mathcal{N}_{2}(E) & \sim Q_{2}(E) & \text { if } & Q_{2}(E) \gg 1 \\
\sim 0 & \text { if } & Q_{2}(E) \ll 1 .
\end{array}
$$

Now that $\mathcal{N}_{2}(E)$ is known, we know the entropy $S(E)$ for any energy $E$ and therefore the model is solved [3] :

$$
\frac{1}{N} S(E)=\lim _{N \rightarrow \infty} \frac{1}{N} \log \left(\mathcal{N}_{2}(E)\right)
$$

The result is

where

$$
\begin{aligned}
\frac{1}{N} S(E) & =\psi(E) \text { if } \psi(E)>0 \\
& =-\infty \text { if } \psi(E)<0
\end{aligned}
$$

$$
\psi(E)=\max _{\substack{\varepsilon \\|\varepsilon|<N J \sqrt{a_{1} \log \alpha_{1}}}}\left[\log \alpha_{1}+\log \alpha_{2}-\frac{1}{a_{1}}\left(\frac{\varepsilon}{N J}\right)^{2}-\frac{1}{a_{2}}\left(\frac{E-\varepsilon}{N J}\right)^{2}\right] .
$$

Note that $S(E)=-\infty$ means only that there is no configuration at energy $E$.

We see clearly that there are 2 possible important energies : the energy $E^{\prime}$ where $\psi(E)$ vanishes and the energy $E^{\prime \prime}$ where the value of $|\varepsilon|$ which gives the maximum in (25) crosses $N J \sqrt{a_{1} \log \alpha_{1}}$. Depending whether $\left|E^{\prime}\right|>\left|E^{\prime \prime}\right|$ or $\left|E^{\prime}\right|<\left|E^{\prime \prime}\right|$, the model has different behaviours. Let me just give here the result of this rather simple discussion. One has two cases.

In the first case defined by

$$
\frac{a_{1}}{\log \alpha_{1}}>\frac{a_{2}}{\log \alpha_{2}}
$$

One finds that the model exhibits two phase transitions at two temperatures

$$
T_{1}=\frac{J}{2}\left(\frac{a_{1}}{\log \alpha_{1}}\right)^{1 / 2} \text { and } T_{2}=\frac{J}{2}\left(\frac{a_{2}}{\log \alpha_{2}}\right)^{1 / 2}
$$

and the free energy is given by

$$
\begin{aligned}
& \begin{array}{ll}
\frac{1}{N} \overline{\log Z}=\log 2+\frac{J^{2}}{4 T^{2}} & \text { if } T>T_{1}
\end{array} \\
& =\log \alpha_{2}+\frac{a_{2} J^{2}}{4 T^{2}}+\frac{J}{T} \sqrt{a_{1} \log \alpha_{1}} \quad \text { if } \quad T_{1}>T>T_{2} \\
& =\frac{J}{T}\left(\sqrt{a_{1} \log \alpha_{1}}+\sqrt{a_{2} \log \alpha_{2}}\right) \quad \text { if } \quad T_{2}>T \text {. }
\end{aligned}
$$


The physical interpretation of these 2 transitions is rather obvious. Above $T_{1}$, there is no group which has a finite Boltzmann weight [8-10]. At $T_{1}$, the system chooses the few groups which have the lowest energies $\varepsilon$. For $T_{1}<T<T_{2}$ the system is frozen in a few groups but inside each group it looks as if it was in its high temperature phase. At $T=T_{2}$, the system becomes completely frozen at its ground state energy. For $T<T_{2}$, there are only a few configurations in each of the few groups which have a finite weight.

The second case is defined by

$$
\frac{a_{1}}{\log \alpha_{1}}<\frac{a_{2}}{\log \alpha_{2}}
$$

In this case, one finds that this model has exactly the same free energy as the RE model

$$
\begin{aligned}
\frac{1}{N} \overline{\log Z} & =\log 2+\frac{J^{2}}{4 T^{2}} \quad \text { if } \quad T>\frac{J}{2 \sqrt{\log 2}}=T_{\mathrm{c}} \\
& =\frac{J \sqrt{\log 2}}{T} \quad \text { if } T<T_{\mathrm{c}} .
\end{aligned}
$$

This case is also interesting because it shows that, if the distances in energy between groups (which play in the GREM the role of valleys) are too small, then the first phase transition disappears.

It is clear that the generalization to any finite $n$ is straightforward. The discussion will become more complicated because at each level there are more cases to consider but there is no difficulty at all to do it for any finite $n$. I believe that for some families of $a_{i}$ and $\alpha_{i}$, the solution can be done easily for any $n$ (for example when the ratio $a_{i} / \log \alpha_{i}$ is a monotonic decreasing function of $i$ ). The physical result will always be that there are a finite number of transitions. In the limit $n \rightarrow \infty$, the spin glass phase will be the region where these transition temperatures become dense.

At the end of this Letter, I would like to mention a few questions which would be worth studying by means of this Generalized Random Energy Model :

1) the first thing to do of course is to solve the GREM for $n>2$;

2) one can try to write renormalization equations which relate what happens at level $i$ to the properties of level $i-1$ (in the hierarchy);

3) one can try to solve the GREM for which $u$ and $v$ are given by (14) and (15) and see whether it gives correctly the beginning of the expansion for $p \rightarrow \infty$ of the $p$ spin glass models;

4) one should try to calculate the 3 configuration correlations $P\left(E_{1}, E_{2}, E_{3}\right)$ of the GREM and compare them with those of other spin glass models;

5) one can try to define spin models which give the GREM in some limit. This would allow the calculation Parisi's $q(x)$ [5];

6) one can try to introduce a magnetic field as was done in the RE model [4];

7) one can try to calculate $P\left(E_{1}, E_{2}\right)$ for finite dimensional lattices and see how the RE approximation [4] can be improved by taking into account these correlations ;

8) one can study non self averaging effects [8-10];

9) one can try to generalize the GREM again in order to treat the correlations between energies of 3 configurations.

\section{Acknowledgments.}

It is a great pleasure to thank E. Gardner, H. J. Hilhorst and G. Toulouse for numerous stimulating discussions and for their encouragement. 


\section{References}

[1] See e.g. Proceedings of the Heidelberg Conference (1983) J. L. Van Hemmen and R. Morgenstern eds. (Springer Verlag) 1983.

[2] Sherrington, D. and KirkPatrick, S., Phys. Rev. Lett. 35 (1975) 1972.

[3] Derrida, B., Phys. Rev. Lett. 45 (1980) 79.

[4] Derrida, B., Phys. Rev. B 24 (1981) 2613.

[5] Gross, D. and MeZard, M., Nucl. Phys. B 240 (FS12) (1984) 431.

[6] Eisele, Th., Commun. Math. Phys. 90 (1983) 125.

[7] Olivieri, E. and Picco, P., Commun. Math. Phys. 96 (1984) 125.

[8] Derrida, B. and Toulouse, G., J. Physique Lett. 46 (1985) L-223.

[9] Mezard, M., Parisi, G., Sourlas, N., Toulouse, G. and Virasoro, M., Phys. Rev. Lett. 52 (1984) 1146 and J. Physique 45 (1984) 843.

[10] Mezard, M., Parisi, G. and Virasoro, M., J. Physique Lett. 46 (1985) L-217. 